\title{
"Études Littéraires. Théories, analyses et débats», sous la direction de Christiane Ndiaye
}

Alba Pessini

\section{(2) OpenEdition}

1 Journals

\section{Édition électronique}

URL : https://journals.openedition.org/studifrancesi/38462

DOI : 10.4000/studifrancesi.38462

ISSN : 2421-5856

Éditeur

Rosenberg \& Sellier

\section{Édition imprimée}

Date de publication : 15 décembre 2004

Pagination : 660-662

ISSN : 0039-2944

\section{Référence électronique}

Alba Pessini, « «Études Littéraires. Théories, analyses et débats», sous la direction de Christiane Ndiaye », Studi Francesi [En ligne], 144 (XLVIII | III) | 2004, mis en ligne le 30 novembre 2015, consulté le 08 mai 2021. URL : http://journals.openedition.org/studifrancesi/38462 ; DOI : https://doi.org/ 10.4000/studifrancesi.38462

Ce document a été généré automatiquement le 8 mai 2021.

\section{(c) $(1) \ominus$}

Studi Francesi è distribuita con Licenza Creative Commons Attribuzione - Non commerciale - Non opere derivate 4.0 Internazionale. 


\title{
«Études Littéraires. Théories, analyses et débats», sous la direction de Christiane Ndiaye
}

\author{
Alba Pessini
}

\section{RÉFÉRENCE}

«Études Littéraires. Théories, analyses et débats», numéro consacré à Émile Ollivier sous la direction de Christiane NDIAYE, vol. 34, n³, 2002, pp. 133.

1 Le numéro 34 des «Études Littéraires» réunit des travaux consacrés à l'œuvre d'Émile Ollivier et chaque étude s'attache à nous dévoiler un ou plusieurs traits de cette production encore trop peu étudiée de façon approfondie malgré son indéniable mérite littéraire et les nombreux prix qui l'ont couronnée. Les auteurs de ces essais, qui sont parmi les critiques les plus attentifs de la littérature haïtienne, tentent ainsi de combler une lacune et de rendre hommage à l'écrivain qui nous a quitté au mois de novembre 2002. Dans son étude Émile Ollivier: enracinerrant de Notre-Dame-de-Grâce, Thomas Spear se penche sur l'ensemble de l'œuvre d'ollivier et illustre comment les deux productions, celle du professeur et celle de l'écrivain, s'éclairent mutuellement et cheminent en parallèle tout en gardant leur spécificité. Si le langage est différent selon qu'Ollivier s'adresse à un public de spécialistes ou à un lecteur de romans, les thèmes abordés se recoupent. Nous retrouvons les sujets chers au sociologue et à l'homme de lettres: le drame des naufragés, des boat-people qui perdent leur vie pour un rêve avorté mais aussi Haïti qui devient «une poubelle pour les déchets toxiques des Américains». L'engagement d'ollivier se manifeste dans ses fictions romanesques quand il s'attache à décrire la société haïtienne, mais ce souci de vérité semble s'estomper au fil des romans jusqu'à Mille Eaux où les rues de Port-au-Prince "grouillent plutôt de splendeurs agréables». Spear souligne toute l'importance que revêt chez Ollivier le mélange d'Histoire et de fiction, en particulier dans le roman Mère-Solitude (1983) où la recherche du passé, de l'histoire familiale du jeune Narcès s'enchevêtre 
avec l'Histoire de la terre haïtienne, de sa décadence et de son «délabrement». Parmi les différents adjectifs tels que migrant ou marginal employés par Spear pour qualifier Émile Ollivier, il en est un, enracinerrant, que le critique emprunte à l'écrivain JeanClaude Charles et qui semble lui convenir mieux que les autres. En effet, il insiste ainsi sur une des caractéristiques de l'écrivain qui tout au long de son parcours de création interpelle à la fois la racine, le pays de la mémoire, les origines mais aussi «les réalités nouvelles de la migration». À ce propos son dernier recueil de nouvelles Regarde, regarde les lions s'approprie des deux espaces; l'auteur campe ses personnages tantôt en Haïti, tantôt à Montréal, deux mondes qui désormais lui appartiennent et où il semble avoir trouvé sa place, dans un entre-deux qui est emblématique d'un parcours, d'un auteur «qui navigue, qui fraye un passage entre l'écriture scientifique et l'écriture d'imaginaire, entre la fiction et la réalité, entre le Québec et Haïti».

2 Le transculturel interpelle Éloïse Brière dans son essai Quand Erzulie investit l'espace de Maria Chapdelaine. Son propos met l'accent sur l'évolution de la littérature québécoise des vingt dernières années qui s'est ouverte aux apports extérieurs, «ces fibres venues d'ailleurs s'entremêlent à la «pure laine» québécoise, produisant un nouveau tissu, une littérature autre, où les éléments culturels du Sud entrent dans la ronde postmoderne de la métropole montréalaise». Le critique analyse dans cette perspective le dernier recueil d'Émile Ollivier, publié en 2001, et souligne la différence qui s'impose avec la première œuvre datant de 1977, Paysage de l'aveugle. Si les espaces proposés, Haïti et le Québec, sont bien les mêmes, le narrateur accomplit un cheminement au cours duquel il va subir une profonde transformation, il est au terme du parcours "d'une autre facture [...], ni pure laine, ni pure tropicalité». Une nouvelle figure narrative se dessine donc et offre au lecteur sa double appartenance, néo-québécoise et haïtienne capable de traduire, de rendre lisible l'haïtianité qui a posé ses empreintes dans la littérature québécoise. Émile Ollivier, nous dit Éloïse Brière, rassemble différents éléments, différents codes qu'il faut déchiffrer, construit un nouvel espace que tous les personnages n'arrivent pas à habiter comme Lafcadio, le protagoniste de la nouvelle Une nuit, un taxi, dont la vie se perd la nuit de la Saint-Jean lors de sa rencontre avec la déesse vaudou Erzulie, venue de loin lui rendre visite au Canada pour lui rappeler sa dette envers les loas. Le narrateur de la nouvelle sert de pont, de passeur culturel, il déchiffre et rend accessible l'incompréhensible, l'inexplicable et a "la tâche de départager les fantômes des faits dans un récit permettant aux autorités de comprendre ce qui leur est foncièrement étranger», un narrateur dont l'identité se confond évidemment avec celle de l'écrivain qui s'acharne, dit-il, à "améliorer la lisibilité du monde".

3 Yves Chemla lui, s'interroge dans On ne revient pas indemne de l'étonnement haïtien sur un des aspects qui est au cœur du récit autobiographique Mille Eaux. La figure de la faille, qui se manifeste sur des plans différents et traverse tout le texte: la fissure familiale d'abord qui concerne de façon traditionnelle non seulement le père et la mère, chacun voué à un destin indépendant - la mort pour l'un, la folie pour l'autre - mais aussi la famille plus élargie des nombreux frères et sœurs du protagoniste Milo (Émile Ollivier) qui ne feront dans sa vie qu'une apparition fugace lors de l'enterrement du père. Les failles selon Chemla se multiplient, à la fissure sociale représentée par les déménagements successifs de la mère et par les choix existentiels de personnages secondaires comme celui de l'exil, s'ajoute aussi la fissure historique. Dans son inventaire des brisures qui blessent à la fois l'existence individuelle et l'histoire 
collective, Yves Chemla souligne aussi l'importance d'une écriture qui quand elle se détache des contingences «redevient lyrique [et] l'image du paradis alors ressurgit, mais un paradis des sens, un paradis dans lequel le corps caresse enfin la présence au monde en s'y laissant caresser».

4 Le texte de Joëlle Vitiello Haïti dans l'oeuvre d'Émile Ollivier se propose d'analyser les différentes perceptions de l'île natale dans les romans. Le départ d'Haïti, les nombreuses années passées en terre étrangère vont conduire l'auteur à porter un regard particulier sur son lieu de naissance et c'est «la distance qui permet l'émergence d'un style exprimant l'essentiel de l'île natale, qui touche à celle-ci au plus près, et qui colle au corps, au texte, sans jamais épuiser les possibilités de la représenter». Retrouver l'île équivaut à la reconstruire à travers la mémoire, à travers une quête généalogique qui s'opère dans bon nombre de romans comme Mère-Solitude, Les Urnes scellées ou dans Mille Eaux. Joëlle Vitiello souligne surtout la présence dans ces textes de personnages qui se prêtent à des métiers d'excavations - physique ou dans le temps comme l'ethnologue, le paléontologue, l'archéologue, l'archiviste. La tâche de l'écrivain ressemble curieusement aux ouvrages de ces hommes qui interrogent les restes et les traces. L'exil et ses nombreuses facettes, les sentiments qui y sont étroitement liés comme la nostalgie, le rejet, la tentation et l'impossibilité du retour sont au centre des travaux de fouille menés par ollivier et ils déterminent une multiplicité de visions de la terre natale. Les personnages, qu'ils s'appellent Normand Malavy, Brigitte Kadmon ou Adrien Gorfoux, véhiculent une mémoire différente du lieu et c'est dans ce kaléidoscope de récits qui mêlent les histoires individuelles à l'Histoire collective, les espaces de l'enfance et de l'exil, la mémoire et le souvenir qu'Ollivier s'exerce comme un artisan.

5 Dans Le baroque au féminin Christiane Ndiaye, qui a dirigé l'ensemble de ce dossier et en a rédigé la présentation, nous invite à la recherche des éléments baroques chez les personnages féminins de Mère-Solitude et des Urnes scellées. Mais sa réflexion ne se limite pas aux textes d'ollivier, elle met en évidence l'évolution du roman haïtien qui semble s'éloigner de l'esthétique du réel merveilleux pour interpeller un néo-baroque sur lequel s'interrogent désormais les critiques. Elle souligne l'écart entre la représentation du féminin chez les auteurs qui se réclament du réel merveilleux tels qu'Alexis, Roumain et Depestre et cette même représentation chez Ollivier. Chez les premiers, la femme est porteuse de vie, d'espoir et d'énergie alors que les protagonistes féminines d'ollivier doivent plutôt affronter l'amertume de la vie. Les deux esthétiques offrent, selon Christiane Ndiaye, deux mondes aux caractéristiques divergentes malgré quelques traits communs (goût de la théâtralité, phénomène de métamorphose). Les textes qui se réclament du réalisme merveilleux représentent un univers qui rassure, qui a une logique interne alors que les romans d'ollivier sont le miroir d'un monde symbolisé par l'image du labyrinthe où il n'y a plus de sortie possible, un univers incertain qui accumule, qui se donne en spectacle mais dont les nombreux questionnements restent sans réponse, comme les quêtes d'Adrien dans Les Urnes scellées et de Narcès dans Mère-Solitude pour ne citer que les plus exemplaires.

6 Le baroque est aussi au centre de l'étude de Joubert Satyre, auteur entre autre d'une thèse intitulée Le baroque dans l'œuvre romanesque d'Émile Ollivier, sous l'un de ses traits les plus marquants: la théâtralité et son corollaire, l'ostentation. Joubert relève l'importance du langage théâtral et les différentes formes d'ostentation dans MèreSolitude. Ostentation liée au pouvoir et à son exercice qui se manifeste au niveau 
individuel et collectif, un pouvoir qui cherche à éblouir, qui exhibe sa puissance pour épater et cacher ainsi son inconsistance. L'ostentation trouve sa demeure idéale dans la description de la maison de la famille des Morelli et atteint son paroxysme pour Joubert dans la représentation carnavalesque, «fête baroque par excellence», qui traduit la nature paradoxale du baroque, en effet «le carnaval est un masque qui donne autre chose à voir que ce qu'il montre, qui, paradoxalement cache la vérité pour mieux la montrer». L'auteur de l'étude montre comment ce recours d'ollivier à la théâtralité et à l'ostentation pour décrire les individus et les choses est une façon de combler le vide de l'existence et de l'être en même temps qu'elle les révèle. Émile Ollivier, dans l'article qui clôt les réflexions sur son œuvre, texte d'une conférence tenue lors d'un colloque sur L'avenir des civilisations: hybridation ou purification, s'interroge sur la mondialisation culturelle qui conduit inévitablement à reconsidérer la vision géographique du lieu habité qui entre en contact avec des réalités et des «contrées diverses». Ce concept remet en discussion les principes d'État-nation, d'identité culturelle sur lesquels l'écrivain mais surtout l'écrivain migrant a son mot à dire. Ce texte d'Ollivier confirme les points de vue de Thomas Spear et d'Éloïse Brière, puisque c'est à la fois le sociologue et l'écrivain qui parlent mais aussi parce que l'auteur cherche à mettre en évidence les stratégies d'écriture de l'écrivain migrant, porteur d'une écriture qui «institue un droit d'être autre, d'ici, d'ailleurs, par delà, en deçà, en devenir». 